\title{
Structural and Functional Neuroimaging of Restricted and Repetitive Behavior in Autism Spectrum Disorder
}

\author{
Jenna M. Traynor and Geoffrey B.C. Hall
}

Department of Psychology, Neuroscience \& Behavior, McMaster University, Psychology Building (PC) Room 307, 1280 Main Street West, Hamilton Ontario L8S 4K1, Canada

\begin{abstract}
A prominent symptom of Autism Spectrum Disorder includes restricted and repetitive behaviours. This symptom has been divided into three subtypes: repetitive motor behaviour, insistence on sameness and circumscribed interests. In the past, the neural correlates of these behaviours have been largely understudied. More recently, neuroimaging studies have pointed to a number of neural networks that may underlay these behaviours. However, results from this work have been varied and remain difficult to integrate. The purpose of this review is to summarize recent neuroimaging studies on restricted and repetitive behaviours in autism, and to provide an organized framework that will permit a clearer understanding of the neural correlates of these behaviours. Using a developmental perspective, this review will identify that there are distinct and overlapping neural networks that are associated with repetitive motor behaviour, insistence on sameness and circumscribed interests. In addition, this review will identify a series of executive and affective function tasks that have proven efficacious in the study of repetitive behaviour.
\end{abstract}

Keywords: Autism spectrum disorder, neuroimaging, repetitive behaviour, repetitive motor behaviour, insistence on sameness, circumscribed interests.

\section{INTRODUCTION}

Autism Spectrum Disorder (ASD) is a neurobiological condition characterized by deficits in social communication and restricted, repetitive behavior (RRB) [1]. It is a complex disorder associated with diverse behavioral symptoms and complex neural substrata. Recently, a growing body of neuroimaging research focused on examining RRB in ASD has emerged. RRB encompasses a broad range of heterogeneous behaviors that have proven difficult to quantify [2]. These behaviours likely reflect complex genetic and environmental interactions across development [3]. Given this heterogeneity, the conceptualization of RRB as one broadly inclusive category has been replaced by a framework that stratifies RRB into three distinct subtypes or factors. These include Repetitive Motor Behavior (RMB), Insistence on Sameness (IS) and Circumscribed Interests (Cl) [4]. These three behavioral subtypes have been determined by factor analyses and independent component analyses of diagnostic measures such as the Autism Diagnostic InterviewRevised (ADI-R) and the Autism Diagnostic Observation Schedule (ADOS) [4-6]. The identification of these subtypes has allowed for a more fine-tuned behavioral profile of ASD. For example, RMB is associated with a distinct motor component [7], and has been described as more rudimentary, or "lower

*Address correspondence to this author at the Department of Psychology, Neuroscience \& Behavior,) McMaster University, Psychology Building (PC) Room 102, 1280 Main Street West, Hamilton Ontario L8S 4K1, Canada; Tel: 905-522-9140 ext. 23033; E-mail: hallg@mcmaster.ca order," in nature [8]. Some examples of RMB include hand flapping, body rocking and other self-stimulatory behaviors. On the other hand, IS and $\mathrm{Cl}$ have been described as "higher order" [8] and are linked to distinctly cognitive components [7]. Rigid adherence to routines and unusually intense preoccupations are examples of IS and $\mathrm{Cl}$, respectively.

Recently, a number of neuroimaging research studies have examined the relationship between these distinct RRB subtypes and underlying neural circuitry [9], bridging the gap between behavioral and neural findings in ASD. However, the results of these studies have been mixed. For example, by using structural magnetic resonance imaging (MRI), some studies have revealed patterns of morphological brain changes that are significantly correlated with different RRB subtypes [10-12], whereas other studies have found that no correlations hold across RRB categories [24]. These studies have been additionally complicated by the use of different neuroimaging methods and sample demographics. For example, in addition to MRI, many studies have used functional magnetic resonance imaging (fMRI) to examine RRB indirectly, by analyzing brain activation during the performance of executive functioning (EF) tasks. These EF tasks have been used as alternative RRB measures, as a means to examine RRB using task-based paradigms [14]. Recently, a number of EF tasks have been proposed to study RRB in ASD. Findings have linked performance and neural activation during these EF tasks to ASD scores on diagnostic measures [15-18]. In addition, a handful of studies have drawn correlations between neural 
activation on some of these EF paradigms and specific RRB scores on diagnostic measures. These studies demonstrate the specific efficacy of these paradigms in the study of RRB. With some studies establishing direct links between RRB and brain function, and other studies proposing new paradigms to investigate RRB in $A S D$, a review and organization of the literature is needed. These findings hold great clinical promise, as they may contribute to a more precise neural understanding of RRB. They also offer potential for the development of targeted interventions and novel pharmacological therapies. With this said, this review has two purposes.

First, this review is intended to synthesize recent structural MRI studies into an organized framework to permit a clearer understanding of the morphological abnormalities associated with RMB, IS and $\mathrm{Cl}$. This summary will demonstrate that these distinct RRB subtypes are associated with both discrete and overlapping regional abnormalities that are best considered from a developmental framework. For example, discrete regional abnormalities of the frontal cortex and cerebellum have been associated with RMB [19]. In contrast, overlapping abnormalities in frontal and striatal regions appear as key contributors to all three subtypes (i.e., RMB, IS and $\mathrm{Cl}$ ) across development [11, 19-20]. This review presents a broad frontal striatal developmental trajectory of RRB in ASD (Figure 1), and indicates underlying motor and cognitive control deficits in the development of RRB.

The second purpose of this review is to synthesize recent $\mathrm{fMRI}$ studies, focusing on network abnormalities and differences in RRB neural circuitry. Inherent in this synthesis is an evaluation of several EF tasks that have been used as RRB proxies in a number of fMRI studies. EF tasks that examine visual motor coordination, motor and cognitive inhibition, rule violation, cognitive set shifting, target detection, and delay incentive as they relate to RRB, are included in this review. An organized framework of EF tasks that have been used as RRB proxies is presented. This framework outlines which EF tasks elicit brain activation, which is correlated with RRB scores on diagnostic measures. This framework will allow for an initial organization of findings and pinpoint areas of interest for future research. This comparison identifies that there are overlapping neural network abnormalities that are elicited by certain EF tasks. This overlap is consistent with theories identifying ASD as a brain connectivity disorder. These findings are presented in a neurocognitive model of RRB (Figure 2), and identify EF tasks that may be of value in future RRB research.

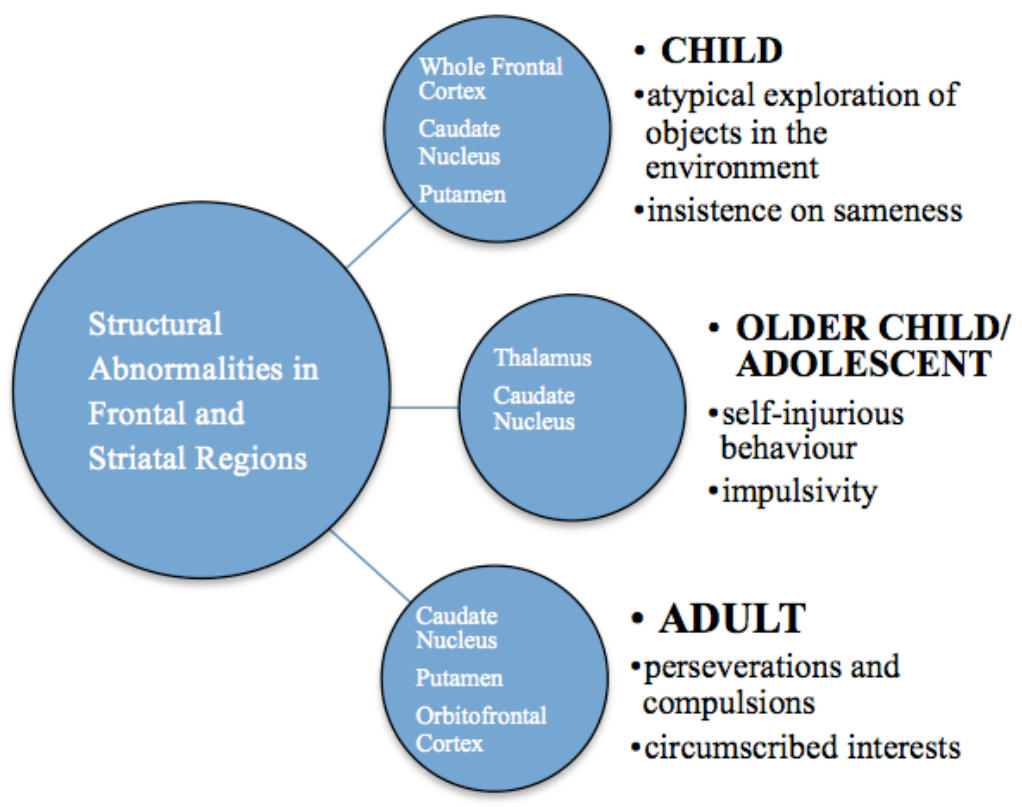

Figure 1: A Frontal and Striatal Developmental Trajectory of RRB in ASD.

From childhood to adulthood, the development of RRB is correlated with several structural abnormalities in frontal and striatal regions. During childhood, enlargement of the entire frontal cortex is correlated with atypical exploration of objects in the environment [19]. Also during childhood, abnormal growth rate of striatal structures (i.e., caudate nucleus and putamen) is correlated with insistence on sameness [9] (top). During older childhood and adolescence, abnormal thalamic volume is correlated with self-injurious behaviour [10] and enlarged caudate nucleus volume is correlated with measures of impulsivity [31] (middle). In adulthood, abnormal caudate nucleus [11-12] and putamen [11] volumes are correlated with perseverative and compulsive behaviour, and orbitofrontal cortex enlargement is correlated with circumscribed interests [20] (bottom). 


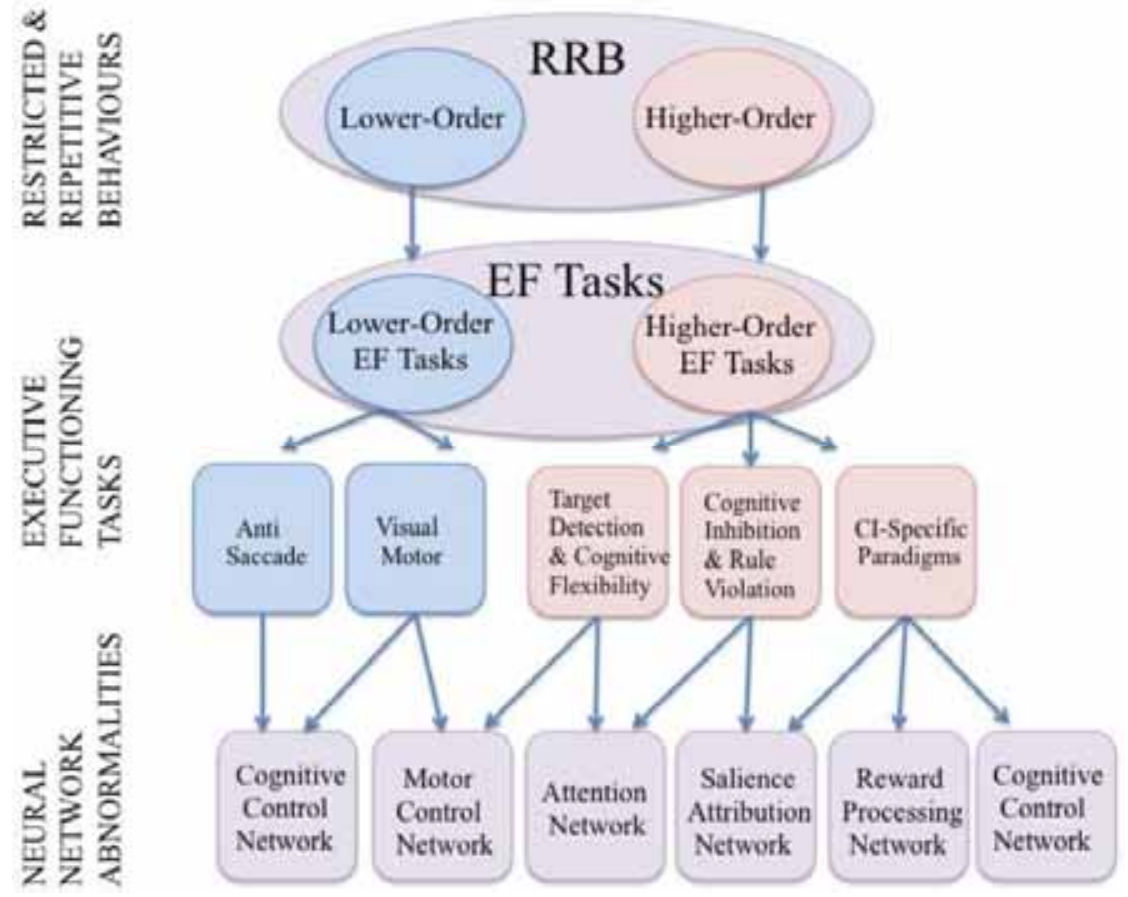

Figure 2: A neurocognitive model of RRB in ASD.

Abnormalities in the cognitive control, motor control, attention, salience attribution, and reward processing networks, are identified during tasks of executive function (EF) employed to study RRB. Moreover, the majority of these network abnormalities appear as dimensional. Several EF tasks reveal abnormal activation in the same neural network. Adapted from findings in [13, 41-45, 47-48, 54-57]. Legend: RRB - Restricted and Repetitive Behaviours. EF Tasks - Executive Function Tasks. Cl-SpecificCircumscribed Interest-Specific.

More specifically, this model shows that abnormalities in neural networks implicated in cognitive and motor control, attention, salience attribution, and reward function, may underlie RRB in ASD. Differences in sample age, diagnosis, and methodology between studies are also discussed in light of their impact on results.

The structure of this review is as follows: first, structural neuroimaging (MRI) studies are discussed. Then, functional neuroimaging (fMRI) studies are examined. This review of fMRI studies includes studies that have employed tasks of EF as indirect measures of RRB. Within each structural and functional section, $\mathrm{RMB}$, IS and $\mathrm{Cl}$ behaviors are discussed. Following this, resting-state functional connectivity and diffusion tensor imaging methods are briefly discussed. Restingstate and diffusion tensor imaging studies are examined separately because they are relatively new approaches to studying RRB in ASD, and do not yet fit the stratified framework of subtypes outlined above. Last, future directions are considered.

\section{METHODOLOGY}

Reported in the current review are all studies that were identified based on a comprehensive literature search in PubMed, PsychINFO and Google Scholar. Searches were conducted for neuroimaging studies conducted between 1999 and 2013 that examined repetitive behaviours in children and adults with an ASD (autism, high functioning autism, Asperger syndrome, and pervasive developmental disorder not otherwise specified). Only peer-reviewed articles for which English text was available were included in the review. Key words used in the literature search included "autism", "neuroimaging", "[functional] magnetic resonance imaging", "diffusion tensor imaging", "restricted [and] repetitive behaviour", "circumscribed interests", "insistence on sameness", "motor stereotypies", "repetitive motor behaviour", "repetitive cognitive behaviour", and "executive function". See Table $\mathbf{1}$ for a list of papers reviewed, including number of participants, age range, diagnosis, type of neuroimaging method used and a summary of findings from each study included in this review.

\section{Structural MRI studies and RRB}

\section{Lower Order, Repetitive Motor Behavior (RMB)}

The importance of studying RMB is underscored by the observation that it is the only RRB subtype that is 
Table 1: Neuroimaging Results Presented in this Review

\begin{tabular}{|c|c|c|c|c|c|}
\hline Author(s) & Sample & $\begin{array}{l}\text { Age Range } \\
\text { (y) }\end{array}$ & Method & Neural region(s) & Results (ASD vs. comparison group) \\
\hline $\begin{array}{l}\text { Langen et al. } \\
\quad(2013)[9]\end{array}$ & $\begin{array}{l}\text { ASD }(n=49) \text { vs. } \\
\operatorname{TD~}(n=37)\end{array}$ & $9-12$ yrs & MRI & $\begin{array}{l}\text { Striatum (caudate } \\
\text { nucleus and } \\
\text { putamen) }\end{array}$ & $\begin{array}{l}\text { Growth rate of striatum correlated with } \\
\text { insistence on sameness behaviour on the } \\
\text { ADI-R at preschool age }\end{array}$ \\
\hline $\begin{array}{l}\text { Duerden et al. } \\
\text { (2013) [10] }\end{array}$ & $\begin{array}{l}\text { ASD }(n=30) \text { vs. } \\
\operatorname{TD}(n=30)\end{array}$ & $7-15 y r s$ & MRI & $\begin{array}{l}\text { Thalamus, superior } \\
\text { parietal lobe, } \\
\text { somatosensory cortex }\end{array}$ & $\begin{array}{l}\text { Volume of thalamus and cortical } \\
\text { thickness of superior parietal and } \\
\text { somatosensory cortex correlated with } \\
\text { self-injurious behaviour }\end{array}$ \\
\hline $\begin{array}{l}\text { Hollander et al. } \\
\text { (2005) [11] }\end{array}$ & $\begin{array}{l}\text { ASD }(n=17) \text { vs. } \\
\text { TD }(n=17)\end{array}$ & $17-57$ yrs & MRI & $\begin{array}{l}\text { Striatum (caudate } \\
\text { nucleus and } \\
\text { putamen) }\end{array}$ & $\begin{array}{c}\text { Enlargement of striatum correlated with } \\
\text { 'obsessions/ compulsions' and } \\
\text { 'perseverations' scores on the ADI }\end{array}$ \\
\hline $\begin{array}{l}\text { Sears et al. } \\
\text { (1999) [12] }\end{array}$ & $\begin{array}{l}\text { ASD }(n=35) \text { vs. } \\
\operatorname{TD}(n=37)\end{array}$ & $12-29 \mathrm{yrs}$ & MRI & Caudate Nucleus & $\begin{array}{l}\text { Enlargement of caudate nucleus } \\
\text { correlated with 'obsessions/ compulsions' } \\
\text { and 'perseverations' scores on the ADI }\end{array}$ \\
\hline $\begin{array}{l}\text { Goldberg et al. } \\
\text { (2011) [13] }\end{array}$ & $\begin{array}{l}\text { HFA }(n=11) \text { vs. } \\
\text { TD }(n=15)\end{array}$ & $8-12$ yrs & $\begin{array}{l}\text { fMRI (using a } \\
\text { Go/No Go task) }\end{array}$ & Insula & Hyperactivation of insula \\
\hline $\begin{array}{c}\text { Pierce \& } \\
\text { Courchesne } \\
\text { (2001) [19] }\end{array}$ & $\begin{array}{l}\text { ASD }(n=14) \text { vs. } \\
\operatorname{TD}(n=14)\end{array}$ & $3-5 y r s$ & MRI & $\begin{array}{l}\text { Frontal cortex } \\
\text { cerebellum }\end{array}$ & $\begin{array}{l}\text { Volume of entire frontal cortex and } \\
\text { cerebellum correlated with the atypical } \\
\text { exploration of objects in the environment }\end{array}$ \\
\hline $\begin{array}{l}\text { Hardan et al. } \\
\text { (2005) [20] }\end{array}$ & $\begin{array}{l}\text { ASD }(n=40) \text { vs. } \\
\operatorname{TD}(n=41)\end{array}$ & $8-46$ yrs & MRI & OFC & $\begin{array}{c}\text { Enlarged OFC correlated with } \\
\text { circumscribed interest scores on the } \\
\text { ADI-R }\end{array}$ \\
\hline $\begin{array}{l}\text { Nordahl et al. } \\
\text { (2007) [23] }\end{array}$ & $\begin{array}{c}\text { Asperger's } \\
(n=15) \text { vs. TD } \\
(n=29)\end{array}$ & $7-18 y r s$ & MRI & Parietal Cortex & $\begin{array}{l}\text { Abnormal parietal folding correlated with } \\
\text { repetitive behaviour scores on the ADI-R }\end{array}$ \\
\hline $\begin{array}{l}\text { Goldman et al. } \\
\qquad \begin{array}{c}(2013) \\
{[24]}\end{array}\end{array}$ & $\begin{array}{l}\operatorname{ASD}(n=31) \text { vs. } \\
\operatorname{TD}(n=30)\end{array}$ & $\begin{array}{l}\text { mean ASD } \\
\text { age } 9 \mathrm{yrs} \\
\text { (range N/A) }\end{array}$ & MRI & $\begin{array}{l}\text { Supplementary motor } \\
\text { cortex, OFC, DLPFC, } \\
\text { ACC, caudate } \\
\text { nucleus, globus } \\
\text { pallidus, thalamus, } \\
\text { hypothalamus }\end{array}$ & $\begin{array}{l}\text { No correlation found between volume of } \\
\text { cortical or subcortical structures and } \\
\text { video coded motor stereotypies }\end{array}$ \\
\hline $\begin{array}{l}\text { Estes et al. } \\
\quad(2011) \\
{[27]}\end{array}$ & $\begin{array}{l}\text { ASD }(n=45) \text { vs. } \\
\operatorname{TD}(n=25)\end{array}$ & $3-4$ yrs & MRI & $\begin{array}{l}\text { Striatum (caudate } \\
\text { nucleus and } \\
\text { putamen) }\end{array}$ & $\begin{array}{l}\text { No significant correlation found between } \\
\text { uncorrected striatal enlargement and } \\
\text { repetitive behaviour scores on the ADOS }\end{array}$ \\
\hline $\begin{array}{l}\text { Langen et al. } \\
\qquad(2009) \\
{[28]}\end{array}$ & $\begin{array}{l}\text { HFA }(n=99) \text { vs. } \\
\text { TD }(n=89)\end{array}$ & $6-25$ yrs & MRI & Caudate nucleus & $\begin{array}{l}\text { Increased growth rate of the caudate } \\
\text { nucleus correlated with insistence on } \\
\text { sameness scores on the ADI-R }\end{array}$ \\
\hline $\begin{array}{l}\text { Langen et al. } \\
\text { (2007) [29] }\end{array}$ & $\begin{array}{l}\operatorname{ASD}(n=21) \text { vs. } \\
\operatorname{TD}(n=21)\end{array}$ & $7-14$ yrs & MRI & Caudate nucleus & Increased volume of caudate nucleus \\
\hline $\begin{array}{l}\text { Herbert et al. } \\
(2003)[30]\end{array}$ & $\begin{array}{l}\text { ASD }(n=17) \text { vs. } \\
\operatorname{TD}(n=15)\end{array}$ & $7-11$ yrs & MRI & $\begin{array}{l}\text { Putamen, globus } \\
\text { pallidus }\end{array}$ & $\begin{array}{l}\text { Increased volume of putamen and globus } \\
\text { pallidus }\end{array}$ \\
\hline $\begin{array}{l}\text { Voelbel et al. } \\
(2006)[31]\end{array}$ & $\begin{array}{l}\text { ASD }(n=38) \text { vs. } \\
\operatorname{TD}(n=13)\end{array}$ & $7-13$ yrs & MRI & Caudate nucleus & $\begin{array}{l}\text { Increased volume of caudate nucleus } \\
\text { predicted measures of impulsivity on the } \\
\text { CPT }\end{array}$ \\
\hline $\begin{array}{l}\text { Agam et al. } \\
(2010)[41]\end{array}$ & $\begin{array}{l}\text { ASD }(n=11) \text { vs. } \\
\operatorname{TD}(n=14)\end{array}$ & $18-38$ yrs & $\begin{array}{l}\text { fMRI (using an } \\
\text { anti-saccade } \\
\text { task) }\end{array}$ & FEF, dorsal ACC & $\begin{array}{c}\text { Hypoactivation of the FEF and dorsal } \\
\text { ACC, reduced functional connectivity } \\
\text { between the FEF and dorsal ACC, } \\
\text { functional connectivity between the FEF } \\
\text { and dorsal ACC correlated with repetitive } \\
\text { behaviour scores on the ADI-R }\end{array}$ \\
\hline $\begin{array}{l}\text { Kana et al. } \\
(2007)[42]\end{array}$ & $\begin{array}{l}\text { HFA }(n=12) \text { vs. } \\
\text { TD }(n=12)\end{array}$ & $19-33$ yrs & $\begin{array}{l}\text { fMRI (using an } \\
\mathrm{N} \text {-back } \\
\text { inhibition task) }\end{array}$ & $\mathrm{ACC}$ & $\begin{array}{c}\text { Hypoactivation of the ACC, atypical } \\
\text { connectivity of the insula with the frontal } \\
\text { cortex, reduced synchronization and } \\
\text { hypoconnectivity in the frontoparietal } \\
\text { network }\end{array}$ \\
\hline $\begin{array}{l}\text { Schmitz et al. } \\
\text { (2006) [43] }\end{array}$ & $\begin{array}{l}\text { ASD }(n=10) \text { vs. } \\
\operatorname{TD}(n=12)\end{array}$ & $18-52$ yrs & $\begin{array}{l}\text { fMRI (using a } \\
\text { Go/ No Go, a } \\
\text { spatial Stroop, } \\
\text { and a Switch } \\
\text { Task) }\end{array}$ & $\begin{array}{c}\text { Inferior and } \\
\text { orbitofrontal gyrii, } \\
\text { insula, parietal cortex }\end{array}$ & $\begin{array}{l}\text { Hyperactivation of the inferior and } \\
\text { orbitofrontal gyrii, hyperactivation of the } \\
\text { insula and of the parietal cortices }\end{array}$ \\
\hline
\end{tabular}


(Table 1). Continued.

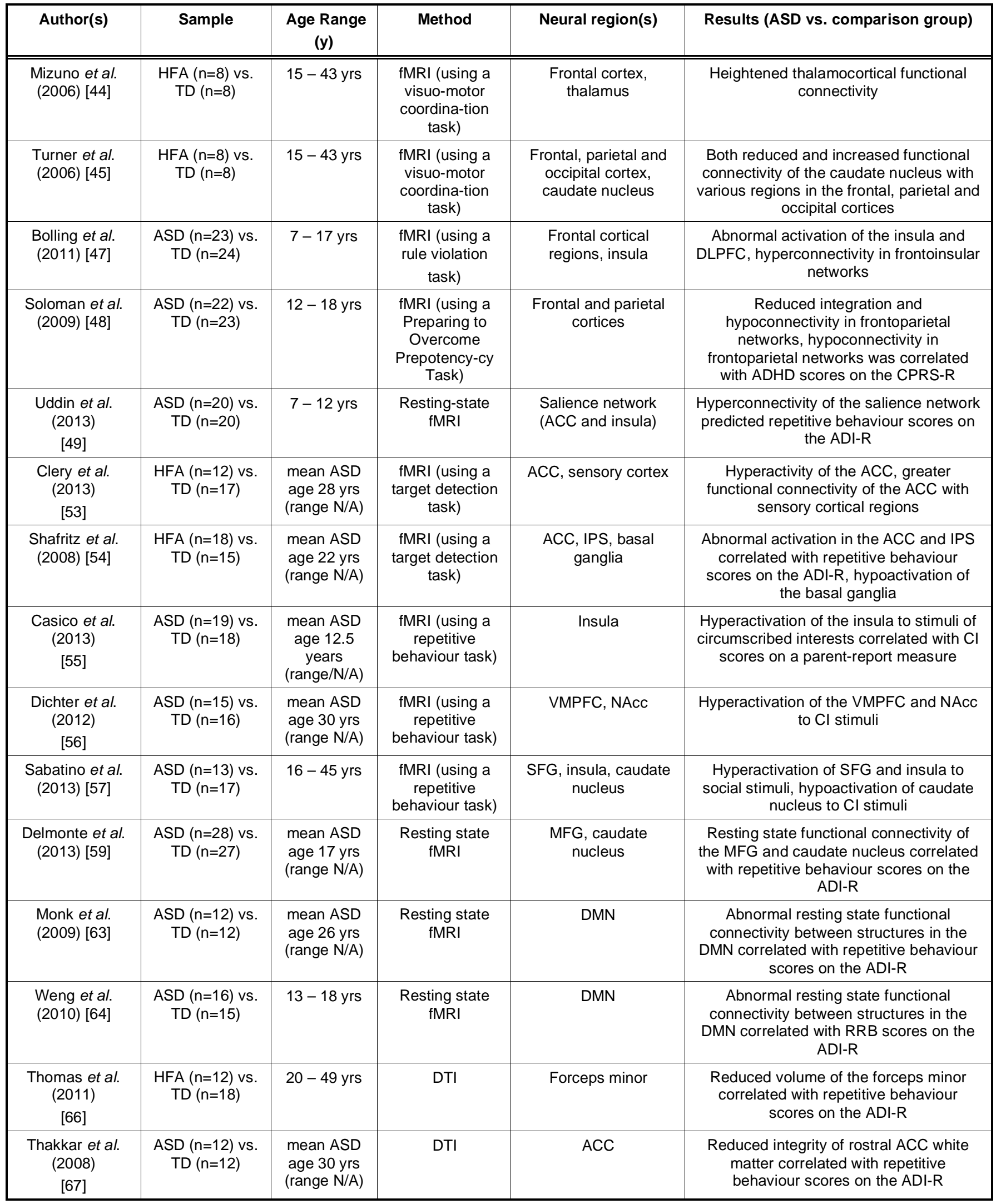

Legend: HFA - high functioning autism, TD - typically developing controls, ADI-R - Autism Diagnostic Interview Revised, CI - circumscribed interests, CPT Continuous Performance Task, ADHD - Attention Deficit Hyperactivity Disorder, CPRS-R - Connor's Parent Rating Scale - Revised, DMN - Default Mode Network, OFC - orbitofrontal cortex, DLPFC - dorsolateral prefrontal cortex, VMPFC - ventromedial prefrontal cortex, SFG - superior frontal gyrus, MFG - medial frontal gyrus, ACC - anterior cingulate cortex, IPS - intraparietal sulcus, NAcc - nucleus accumbens. 
present as early as 15 months [21], and observed across different levels of functioning across the autism spectrum [22]. The expression of RMB is also quite heterogeneous and includes abnormal gait, somatic movement, and vocal production. This heterogeneity suggests potentially widespread early neural candidates. Therefore, the study of neural circuitry underlying RMB offers potential for the identification of early biological markers of ASD that may serve as the initial basis for a cascade of further developmental deficits.

However, structural neuroimaging studies investigating lower order RMB in ASD have been few. One factor that may contribute to this paucity of imaging studies is the requirement for minimized motion while in the MRI scanner. This requirement conflicts with the excessive somatic movement characteristic of RMB, rendering this subtype particularly difficult to investigate using MRI.

Currently, the few available studies relating structural brain abnormalities to RMB show little consensus in results. Pediatric studies have linked increases in entire frontal cortex volume and cerebellar hypoplasia with the atypical exploration of objects in the environment [19]. In addition, self-injurious behaviors have been negatively correlated with thalamic volume, as well as with superior parietal and somatosensory cortical thickness [10]. Abnormal parietal folding has also been linked with measures of RRB in a sample of children with Asperger's Syndrome. Interestingly, this correlation did not hold when examining both high and low-functioning autism samples [23]. This suggests that the biological bases of RRB may be diagnosis dependent. Finally, in contrast to the above findings, a recent study failed to find any significant association between morphometric alterations in child ASD brains and RMB scores [24]. Clearly more work is needed to identify the relation between structural brain abnormalities in childhood ASD and RMB.

\section{Higher Order, Insistence on Sameness, and Circumscribed Interests}

In addition to lower order behaviors (i.e., RMB), research has also been conducted on higher-order behaviors such as IS and $\mathrm{Cl}$. These higher-order subtypes have been studied more extensively, and structural MRI research has revealed substantial cortical and subcortical associations.

Thus far, evidence from structural MRI studies investigating IS and $\mathrm{Cl}$ behaviors suggest that the neural underpinnings of these two subtypes are not completely separable, and may overlap considerably. In addition, data collected from pediatric and adult samples has revealed an early developmental progression of broad frontal and striatal involvement in these higher-order behaviors [9, 11, 19-20].

Although it has been found that higher order IS and $\mathrm{Cl}$ exist in very young ASD samples [25-26], associations between these subtypes and striatal abnormalities show a complex pattern across development. For example, in 3-4 year old ASD samples, findings of uncorrected striatal enlargement in ASD children relative to controls have failed to meet statistical significance [27]. However, longitudinal data have revealed faster striatal growth rate (as opposed to enlargement) in school aged ASD subjects relative to controls [9]. Further, this increased growth rate has been both positively and negatively correlated with IS scores on the Autism Diagnostic Interview - Revised (ADI-R) $[9,28]$. In this age group specifically, increased growth rate of the caudate nucleus has shown a significant negative association with IS [28]. In the same age group, growth rate of the putamen has shown a significant positive correlation with IS [9]. In studies employing older ASD samples between 7 and 12 years of age, findings of increased caudate [29], putamen and globus pallidus [30] volumes relative to controls, have been reported, and increased volume of the caudate has been correlated with measures of impulsivity [31]. These data suggest that abnormal growth rate of the striatum in early childhood may be a precipitating factor in the development of higher-order RRB later in life. As well, enlargement of striatal structures in later childhood and adolescence have been found. However, associations between striatal enlargement and RRB have yet to emerge in this age group.

Continuing this developmental trajectory of the striatum, several adult studies have reported significant correlations between striatal enlargement and higherorder RRB scores. For example, caudate [11-12] and putamen enlargement [11] have been correlated with "perseverations" and "obsessions/compulsions" scores on the Autism Diagnostic Interview. Similar to pediatric findings, these correlations have also been both positive and negative in direction, speaking to the complexity of IS and $\mathrm{Cl}$ across development. Additionally, enlargement of the lateral orbitofrontal cortex (OFC) has been positively correlated with $\mathrm{Cl}$ in adults, but not in children [20]. Complementing this correlational pattern, behavioral research on 
adolescent ASD subjects has demonstrated that as age increases, the severity of $\mathrm{Cl}$ behavior becomes more intense [32].

\section{Structural MRI and RRB Subtypes - a Summary and Comparison}

Thus far, data on lower order RMB point to enlargement of frontal cortical structures, as well as cerebellar hypoplasia in childhood. In addition, reduced cortical thickness and abnormal folding in post central, parietal and subcortical structures, has been found. However, greater methodological consistency is needed in future RMB studies to substantiate and replicate these findings, as studies have also demonstrated null findings [24]. Given that RMB is of the earliest subtype to occur, findings could aid as structural biomarkers for high-risk ASD children. Taken together, the above data indicate widespread structural abnormality in RMB. The findings are so varied, that it may be of value to further parse these qualitatively different motor behaviors in order to arrive at more discernable RMB subtypes.

In contrast, IS and $\mathrm{Cl}$ subtypes involve irregularities of the OFC and striatum (i.e., putamen and caudate). The OFC plays a role in reward-based decision making, reinforcement learning, and emotion processing [33]. Irregularities of OFC function are also found in obsessive-compulsive disorder [34] indicating that OFC abnormality may broadly underlie narrowranged perseverative behaviour, across diagnostic categories. In addition, the putamen and caudate together form the dorsal striatum, which is implicated in a wide range of functions including inhibition, motor initiation [35-36], salience assessment and reward expectancy in decision making [37]. Taken together, it is suggested that the structural brain regions underlying IS and $\mathrm{Cl}$ stem from frontal-dorsal striatal abnormalities, and that these abnormalities may be associated with deficits in both reward-based or emotional decision making, as well as motor inhibition.

Collectively, an examination of the regions putatively implicated in lower and higher order RRB suggests that there are commonalities in frontal-striatal irregularities across all three subtypes (i.e., RMB, IS, and $\mathrm{Cl}$ ). Moreover, abnormalities in specific structures of the frontal-striatal pathway show associations with specific types of RRB at different developmental time points (Figure 1).

Concerning frontal cortical areas, both $\mathrm{RMB}$ and $\mathrm{Cl}$ are reflected by enlargement of the frontal cortex, with
$\mathrm{Cl}$ behavior specifically localized to abnormalities of the OFC. In addition, striatal abnormalities that are localized to the caudate nucleus appear important to higher order RRB. Other parts of the brain that are closely tied to the striatum (i.e., thalamus) are implicated in self-injurious behavior.

Recent research on the role of the striatum in animal and human studies has revealed its importance in both motor control [35-36] and more cognitive, obsessive-compulsive behaviors [38]. The involvement of the striatum in both lower and higher order behaviour suggests that abnormalities in this group of structures may play a role in motor deficits in early childhood (i.e., RMB), which leads to higher-order, cognitive deficits across development (i.e., IS and $\mathrm{Cl}$ ). This literature may suggest that frontal-striatal abnormality serves as an early biomarker for ASD.

\section{Functional MRI and RRB Subtypes}

The structural architecture of the brain plays an important role in the integration of information across functional networks [39]. Thus, a number of other studies have employed functional MRI (fMRI), in addition to morphometric analyses of MRI data. These studies have used fMRI to examine RRB subtypes indirectly, by analyzing brain activation during the performance of affective and executive functioning (EF) tasks. Both traditional $\mathrm{fMRI}$ as well as functional connectivity analyses have been used. $\mathrm{FMRI}$ uses the blood oxygen level dependent (BOLD) signal to identify regional changes in neuronal activity. These changes are associated with the presentation of a particular stimuli or the performance of a task. Functional connectivity analyses examine the synchronous coupling of activity across localized brain regions in order to provide information about functional integration (i.e., networks) [40].

It is important to consider how EF paradigms may be used in neuroimaging studies to evaluate RRB, as a greater number of studies are establishing relationships between RRB subtypes and neural circuitry. To date, a handful of studies have linked neural activity during EF tasks to distinct RRB subtypes. Many studies have also correlated findings of neural abnormality during EF tasks with total RRB scores on the ADI-R. In the following sections, EF tasks that are both rudimentary and/or motor in nature, as well as those that require higher-order, cognitive demand, have been summarized. EF tasks that have recruited similar neural networks are summarized together, and preliminary links to RRB subtypes are presented. 


\section{Lower Order Executive Functioning Tasks and RRB}

A few fMRI studies have employed EF paradigms that are primarily motor in nature, and these paradigms have been used to explore lower-order RRB in ASD. Results have been correlated with total RRB scores on the ADI-R. These studies have been conducted using older adolescent and adult ASD samples, and future studies need to employ younger samples for better generalization of results. EF paradigms using antisaccade and Go/No Go tasks (assessing motor inhibition), as well as simple visual motor coordination tasks (assessing motor planning and coordination), have been employed to explore the neural circuitry related to RRB. The anti-saccade task has revealed hypoactivation in the frontal eye fields and the dorsal anterior cingulate cortex (dACC), in ASD subjects relative to controls. This task has also produced evidence of reduced functional connectivity between these neural regions [41]. However, despite overall hypoactivation in the frontal eye fields in ASD subjects, greater frontal eye field activation during the antisaccade task has been correlated with more severe total RRB scores on the ADI-R [41]. Agam and colleagues have suggested that this correlation reflects greater cognitive effort in ASD subjects, which is required to successfully inhibit motor behavior. Hypoactivation of the ACC has also been shown during other motor inhibition tasks such as the Go/No Go [42]. Go/No Go performance has been associated with hyperactivation in the left inferior and orbital frontal gyrii in ASD subjects relative to controls [43].

Aside from the anti-saccade and Go/No Go tasks, simple visual motor coordination tasks have been employed. These tasks have revealed heightened functional connectivity in ASD across vast thalamocortical and caudate-cortical networks. Accounts of these aberrant cortical-subcortical connectivity patterns suggest there is reduced, early synaptic pruning, abnormal white matter maturation, and thalamic gate dysfunction during development in ASD [44-45]. These vast abnormalities in corticalsubcortical networks may suggest an array of several neural mechanisms that support RRB. Although these studies did not attempt to draw correlations between BOLD activation and RRB scores, the rudimentary and motoric nature of these tasks identify them as plausible RRB proxy measures. The results are distinguished as relevant to stereotypic behavior in ASD [45] indicating that thalamic-cortical and caudate-cortical connectivity should be investigated in future work.
Higher Order Executive Functioning Tasks and RRB

A number of cognitive EF tasks have been employed in order to deepen the understanding of neural involvement in higher order RRB. These EF tasks have investigated BOLD activation during particular cognitive demands. These cognitive demands have been linked to IS and $\mathrm{Cl}$. Some studies have also directly correlated neural activation during these tasks with IS and $\mathrm{Cl}$ scores on the ADI-R. EF tasks requiring cognitive interference inhibition, rule violation processing, simple target detection, and cognitive set shifting have been employed to investigate the neural circuitry of higher order RRB. Novel paradigms specific to $\mathrm{Cl}$ behavior have also been used. These paradigms are examined in the following sections.

\section{Behavioral Inhibition, Rule Violation and the Salience Network}

Behavioral evidence has demonstrated correlations between deficits in inhibition and higher-order RRB scores on diagnostic measures [17, 46]. This evidence has justified using inhibition paradigms in RRB imaging studies. Interestingly, fMRI studies using inhibition, rule violation processing, and error monitoring paradigms have demonstrated significant overlap in neural abnormalities displayed by ASD subjects, compared to controls. Results from these paradigms have revealed abnormal activation of the insula [13, 43], abnormal activation in extensive frontal control regions [42-43, 47], aberrant connectivity of the insulii with frontal structures [42, 47], abnormal activation in the parietal cortex [43], and reduced synchronization and under connectivity in frontal-parietal networks $[42,48]$. In one study, this lack of frontoparietal synchronization was correlated with an increase in attention-deficit hyperactivity symptoms [48]. This correlation suggests that deficits in attention may possibly underlie differences in cognitive performance between ASD and control subjects. Future studies employing these paradigms should investigate the correlation between RRB scores on diagnostic measures and neural activation. These studies would strengthen the understanding of neural contributors to RRB given the behavioral evidence linking inhibition and RRB [17, 46], and the strong neural overlap during inhibition, rule violation, and error monitoring task performance. Further, these studies identify the involvement of the salience network in RRB. The salience network includes the ACC, and the insula [49]. Closely connected with the salience network are neural circuits 
subserving inhibition and attentional processes (including the ACC, cingulate gyrus, insula and parietal regions). The insula plays a role in attributing negative internal emotional states to decision making [50], and strong insular involvement in the above studies is posited to indicate heightened sensitivity to errors/violations in ASD subjects compared to controls $[13,43]$. Also seen in the above studies is strong frontoparietal network involvement. The frontoparietal network plays a large role in attention and the selection of sensory input from the environment [51].

Taken together, these results suggest that ASD deficits in higher-order executive functioning may involve neural abnormalities in salience attribution, inhibition, and attention networks. Future research investigating correlations between these network abnormalities and RRB scores on diagnostic measures will be essential. These studies may solidify connections between RRB subtypes and salience, inhibitory, and attentional processes. Recent ASD research has revealed neural deficits in attentional alerting, orienting, and executive control networks [52]. This further demonstrates that attentional dysfunction may be a core contributor to ASD symptomatology. Additionally, initial confirmation of salience network involvement in RRB comes from a recent resting-state fMRI study. This study found that hyper-connectivity of the salience network in ASD children was specifically predictive of RRB scores on the ADI-R [49].

In addition to the salience network, evidence of frontoparietal (attention) network involvement in RRB has been shown during other types of EF tasks, such as target detection and cognitive flexibility. This work is described in the following section.

\section{Target Detection, Cognitive Flexibility, and the Frontoparietal Network}

In addition to inhibition and rule violation tasks, target detection and cognitive flexibility tasks have revealed abnormal frontoparietal activation in ASD subjects, relative to controls. Justification for the use of cognitive flexibility paradigms comes from behavioral evidence showing a relationship between cognitive flexibility and RRB [16]. Difficulty with change in the environment that is inherent in certain types of RRB (such as IS) [53] also contributes to the face validity of target detection and flexibility tasks as RRB proxies.

Both cognitive flexibility and target detection tasks have shown abnormal patterns of activation in the ACC [53-54], as well as complex parietal dysfunction [43,
54]. These altered patterns of activation in the ACC and parietal lobe during target detection have been negatively correlated with the severity of RRB scores on the ADI-R [54]. This correlation further implicates attentional (i.e., frontoparietal) processes in the maintenance of RRB. Moreover, hypoactivation of the basal ganglia during poor cognitive set shifting performance has been identified in ASD [54].

To summarize, behavioral evidence linking cognitive flexibility and RRB, and correlations between neural activation during target detection performance and RRB scores, indicate that both of these executive functions underlay RRB in ASD, and should be explored further.

\section{Circumscribed Interests}

Lastly, researchers have employed EF paradigms to specifically investigate the higher-order, Cl subtype [55-57]. These novel approaches use fMRI to investigate BOLD activation during the visual presentation of $\mathrm{Cl}$ stimuli. For example, pictures of common $\mathrm{Cl}$ in ASD, such as planes, trains and other mechanical objects, have been used. BOLD activation during the presentation of $\mathrm{Cl}$ stimuli has been compared to activation during the presentation of other types of visual stimuli, such as social images (e.g., faces), or non-Cl stimuli (i.e., control objects or shapes). Results have revealed that ASD individuals show hyper activation to $\mathrm{Cl}$ stimuli, but not to social or non- $\mathrm{Cl}$ stimuli, in the ventromedial prefrontal cortex (VMPFC)- nucleus accumbens (NAcc) reward network [56] and in insular, salience attribution networks [55]. Hyperactivation of the insula to $\mathrm{Cl}$ stimuli in ASD subjects was also correlated with the intensity of everyday $\mathrm{Cl}$ behavior, as recorded in a parent-report measure [55].

Interestingly, one other study used similar $\mathrm{Cl}$ stimuli in an inhibition paradigm, but found hyperactivation of the superior frontal gyrus and right insular cortex to social stimuli, and not to Cl stimuli [57]. This study also found hypoactivation of the caudate nucleus to $\mathrm{Cl}$ stimuli in ASD subjects [57]. Finally, this study found that higher RRB scores in ASD subjects were correlated with a decrease in left inferior and right middle frontal gyrus activation. Although in contrast to the above results of Casico and colleagues [55], these findings have been interpreted as heightened recruitment of cognitive control areas needed for successful inhibition of social stimuli [57]. This suggests that it may be more difficult for those with ASD to inhibit social stimuli, compared to controls [57]. 
Taken together, these results suggest that salience (i.e., insula) and reward network dysfunction (i.e., nucleus accumbens), as well as defective cognitive control (i.e., caudate nucleus), may be involved in the expression of $\mathrm{Cl}$ behavior. However, increased insular activation to both social and $\mathrm{Cl}$ stimuli indicate a complex role of the salience network in $\mathrm{Cl}$ behavior. The overlapping neural activation during the presentation of both social and $\mathrm{Cl}$ stimuli also indicate potential dysfunction of the reward network in both social and repetitive behavior symptoms of ASD. This suggestion parallels ASD studies that have shown early neuronal disorganization as a factor in the later devaluing of social interaction [58]. This suggestion is also similar to studies that have shown reduced activation of the dorsal striatum to social rewards in ASD [59]. Given these findings, future studies should further explore the relationship between social and $\mathrm{RRB}$ deficits, and their underlying neural counterparts.

\section{Executive Functioning Tasks and RRB Subtypes - a Neurocognitive Model}

Taken together, the above affective and executive functioning paradigms have revealed important information about the neural circuitry underlying RRB in ASD. Lower order EF tasks investigating motor inhibition and visual motor coordination have revealed reduced frontal eye field-dACC connectivity. These tasks have also revealed increased connectivity in an array of cortical-subcortical networks. These studies have implicated several neural mechanisms that may underlay lower order RMB, however, future work is needed to solidify connections between specific RMB subtypes and abnormal neural circuitry. Given the vast heterogeneity in RMB across the spectrum, it is likely that the above results may reflect specific forms of $\mathrm{RMB}$, while failing to identify the neural mechanisms of other forms. Alternately, higher order EF paradigms have identified strong frontoinsular and frontoparietal involvement in RRB. In addition, reward network abnormalities, as well as cognitive control deficits, have been shown during performance in $\mathrm{Cl}$-specific paradigms that encompass affective elements.

Collectively, neural abnormalities in the studies discussed above reveal that RRB in ASD may involve particular deficits in cognitive control, motor control, attention, salience attribution, and reward processing. Importantly, overlap in neural circuitry involvement during distinct EF task performance is shown. This underscores the complexity of neural involvement in the broader RRB subtype. This overlap is not surprising. Estimates suggest that between 1 and 6 independent neural components may overlap at a given voxel in the brain [60]. Viewed in this way, RRB in ASD appears as neurocognitively dimensional (Figure 2).

More specifically, abnormalities in a given neural network are identified during performance in multiple EF tasks. For example, abnormalities in the frontoparietal attention network appear as important to consider during target detection, rule violation, cognitive flexibility, and inhibition tasks. This neurocognitive dimensionality in RRB will be important to consider in future work. As there is a lack of intervention practices targeted toward RRB in ASD [61], these findings may provide a foundation for the development of reinforcement-based interventions that target specific neurocognitive deficits. They may also be useful for individuals displaying unique RRB profiles. Additionally, EF paradigms employing antisaccade, target detection, and $\mathrm{Cl}$-specific, affective elements, have demonstrated efficacy in the study of RRB. These paradigms have drawn direct correlations between brain activation and RRB scores. EF paradigms employing cognitive and motor inhibition, rule violation, and cognitive flexibility, also show promise in the study of RRB. Further investigation will be required in order to use these paradigms as evidence-based RRB measures.

\section{Resting-State Functional Connectivity and RRB}

Another imaging method known as resting-state functional connectivity MRI, has revealed an interesting relationship between social communication and RRB symptoms in ASD. Resting state studies (i.e. scans with an absence of any task) make use of spontaneous, low frequency oscillations in the BOLD signal that are correlated across functionally related regions [62]. These studies have identified a relationship between default mode network connectivity and the broader RRB class [63-64]. Interestingly, aberrant resting state connectivity of the posterior cingulate cortex (PCC) with both frontal and temporal cortices, and with the parahippocampal gyrus, has been correlated with poor verbal communication scores, poor social functioning scores, and an increase in RRB scores on the ADI-R [63-64]. These studies suggest a functional overlap between social and RRB symptoms in ASD, which can be observed at a basic, resting-state level. These findings also compliment the results of functional scanning paradigms in the above section (Circumscribed interests) that reveal overlap in neural responses to social and RRB (i.e., Cl) stimuli. 
Aside from the default mode network, one other study also found a positive correlation between right medial frontal gyrus and caudate nucleus resting-state connectivity and RRB scores on the ADI-R [59]. This finding further demonstrates that abnormalities in the frontostriatal system are involved in RRB. Finally, hyper connectivity of the resting-state salience network has been correlated with RRB scores on the ADI-R [49]. In future, continued use of resting-state functional connectivity methods should provide useful information about RRB networks, and begin to draw correlations between specific RRB subtypes and resting-state brain function.

\section{Diffusion Tensor Imaging and RRB}

Finally, Diffusion Tensor Imaging (DTI) is an imaging method that uses the directional diffusion of water molecules in the brain to make inferences about the structural integrity of white matter fibres. Fractional anisotropy (FA) is a quantitative DTI value that identifies the degree of coherence between the direction of water molecules, indicating high or low connectivity of white matter microstructure [65]. Compared to other neuroimaging methods, there has been limited RRB research using DTI. However, there is a need for research using this method because it is important to investigate whether areas of altered brain activation are also associated with altered connectivity and changes to the microstructure of white matter.

DTI studies have identified both cortical and subcortical white matter abnormalities. Reduced volume of the forceps minor - a white matter tract linking the lateral and medial surfaces of the frontal lobes [66] and reduced integrity of rostral ACC white matter [67] have been correlated with RRB scores on the ADI-R. An excess of short-range connections in white matter inferior to the ACC [68] have also been hypothesized to contribute to RRB expression and the inability of individuals with ASD to disengage from a task or stimulus. Abnormalities in white matter surrounding the basal ganglia have also been correlated with behavioral inhibition performance [69]. This provides further support for the use of inhibition EF tasks to explore the architecture underlying RRB.

Altogether, these findings indicate that structural white matter abnormalities may be associated with frontal, cingulate, and striatal alterations in ASD subjects, all of which are implicated in repetitive behavior. Yet, neural patterns associated with specific RRB subtypes are not identified from these DTI studies. Future work would benefit from an approach that stratifies RRB subtypes, and correlates these subtypes with specific white matter abnormalities.

\section{FUTURE DIRECTIONS}

This review has identified that RRB subtypes in ASD encompass both distinct and overlapping neural networks that follow important developmental trajectories. The identification of distinct systems may be useful in the creation of targeted treatments for various individual RRB profiles, as well as in the identification of RRB biomarkers. Overlap in neural circuitry across subtypes is reflective of ASD as a heterogeneous condition, and reflects the complexity of brain-behavior relationships in RRB.

A comparison of the neural networks underlying $\mathrm{RMB}$, IS and $\mathrm{Cl}$ has yielded common frontostriatal structural and functional deficits, implicating motor and cognitive control mechanisms across a broader RRB grouping. Importantly, this review has highlighted an initial developmental trajectory of frontal-striatal morphology and RRB. Although the association between RRB and striatal abnormality is quite complex, early striatal abnormalities may serve as a biomarker for both lower and higher order RRB profiles throughout development. However, correlational findings from studies examining striatal abnormalities and RRB have been largely inconsistent, particularly those examining ASD adolescents. Clearly there are still unknown neural mechanisms supporting higherorder RRB throughout development, and more studies investigating RRB subtypes in adolescent samples are needed. These studies would pinpoint critical periods in development where neural changes shape behavior.

In addition to frontal striatal abnormality, analyses of fMRI data have highlighted potential motor and cognitive control, attention, salience, and reward processing deficits in RRB. Correlations between these neural network abnormalities and RRB scores are informative, and indicate the efficacy of using certain EF tasks to examine RRB networks. Specifically, EF tasks encompassing motor inhibition, target detection, and reward processing, reveal neural activation that is correlated with RRB scores. This demonstrates that these tasks are good RRB proxies.

Additionally, preliminary data has indicated that cognitive flexibility and inhibition tasks, as well as visual motor coordination tasks, should be examined in future work. However, given that the examination of RRB subtypes is a relatively new approach, there is 
significant methodological inconsistency across studies. This makes quantitative comparison between RRB subtypes difficult. Future work would benefit from combining a variety of imaging methods to examine a given RRB subtype. This practice would contribute to more detailed neural profiling of RRB in ASD. For example, data driven or network based analysis methods like Independent Component Analyses could be employed to compliment a general linear model analyses. This would help with further investigation of irregularities in the functional networks underlying RRB.

In addition, an interesting relationship between social and RRB symptoms has emerged, both in taskbased fMRI studies, as well as in resting-state studies. The literature suggests that changes in neural reward function in ASD may play a role in the devaluing of social stimuli, while increasing the salience of $\mathrm{Cl}$ stimuli. Further, abnormal resting state connectivity in overlapping default mode network regions is correlated with both social and RRB symptoms in ASD, indicating that these deficits may share common and intrinsic neural abnormalities.

Finally, both resting-state fMRI and DTI studies provide preliminary evidence for underlying restingstate and white matter abnormalities in RRB, respectively. DTI studies have been important in the confirmation of frontostriatal irregularity in RRB at a white matter level. However, in order to further pinpoint the role of neural deficits in ASD symptoms, future work in these areas should build upon previous studies, and stratify RRB into subtypes. Data driven restingstate studies looking at other large scale resting state networks should also be investigated in the context of RRB. For example, functional abnormalities in thalamocortical networks have been identified during previous task-based paradigms (Mizuno et al., 2006), and future resting-state studies investigating thalamocortical circuitry and RRB could further identify the role of this circuit in RRB subtypes.

Overall, this review has demonstrated the utility of stratifying RRB when conducting neuroimaging research on ASD. By identifying important neural networks that underlie RRB in ASD, this review has aided in the initial neural mapping of symptoms. This work is important as RRB in ASD has traditionally been defined behaviourally. This review has also highlighted affective and executive functioning tasks that can further aid in the study of RRB. In future, this knowledge may be of value in the development of interventions and treatments for individuals displaying unique RRB profiles.

\section{ACKNOWLEDGEMENTS}

The authors would like to thank Samantha Daniel for her generous contributions.

\section{REFERENCES}

[1] American Psychiatric Association. Diagnostic and Statistical Manual of Mental Disorders ( $5^{\text {th }}$ ed.). Washington, DC: Author 2013. http://dx.doi.org/10.1176/appi.books.9780890425596

[2] Langen M, Kas M, Staal W, van Engeland H, Durston S. The neurobiology of repetitive behavior: of mice. Neurosci Biobehav Rev 2010; 35(Pt 3): 345-355. http://dx.doi.org/10.1016/j.neubiorev.2010.02.004

[3] Lewis M, Kim S. The pathophysiology of restricted and repetitive behavior. J Neurodev Disord 2009; 1: 114-132. http://dx.doi.org/10.1007/s11689-009-9019-6

[4] Lam KSL, Bodfish JW, Piven J. Evidence for three subtypes of repetitive behavior in autism that differ in familiality and association with other symptoms. The $\mathrm{J}$ Child Psychol Psychiatry 2008; 49(Pt 11): 1193-1200.

http://dx.doi.org/10.1111/j.1469-7610.2008.01944.x

[5] Cuccaro ML, Shao Y, Grubber J, Slifer M, Wolpert CM, Donnelly SL, et al. Factor analysis of restricted and repetitive behaviors in autism using the Autism Diagnostic Interview-R. Child Psychiatry Hum Dev 2003; 34: 3-17.

[6] Szatmari P, Georgiades S, Bryson S, Zwaigenbaum L, Roberts W, Mahoney W, et al. Investigating the structure of the restricted and repetitive behaviors and interests domain of autism. J Child Psychol Psychiatry 2006; 47(Pt 6): 582590.

http://dx.doi.org/10.1111/j.1469-7610.2005.01537.x

[7] Lewis MH, Tanimura Y, Lee LW, Bodfish J. Animal models of restricted repetitive behavior in autism. Behav Brain Res 2007; 176: 66-74.

http://dx.doi.org/10.1016/j.bbr.2006.08.023

[8] Turner MA. Annotation: repetitive behavior in autism: A review of psychological research. J Child Psychol Psychiatry 1999; 40(Pt 6): 839-849.

[9] Langen $M$, Bos $D$, Noordermeer SD, Nederveen $H$, van Engeland $\mathrm{H}$, Durston $\mathrm{S}$. Changes in the development of the striatum are involved in repetitive behaviors in autism. Biol Psychiatry 2013; 75(Pt 5): 405-411. http://dx.doi.org/10.1016/i.biopsych.2013.08.013

[10] Duerden EG, Card D, Roberts W, Mak-Fan KM, Chakravarty $\mathrm{M}$, Lerch JP, et al. Self-injurious behaviors are associated with alterations in the somatosensory system in children with autism spectrum disorder. Brain Struct Funct 2013; 219(Pt 4), $1251-1261$.

http://dx.doi.org/10.1007/s00429-013-0562-2

[11] Hollander E, Anagnostou E, Chaplin W, Esposito K Haznedar M, Licalzi E, et al. Striatal volumes on magnetic resonance imaging and repetitive behaviors in autism. Biol Psychiatry 2005; 58(Pt 3): 226-232. http://dx.doi.org/10.1016/j.biopsych.2005.03.040

[12] Sears LL, Vest C, Mohammed S, Bailey J, Rason BJ, Piven J. An MRI study of the basal ganglia in autism. Prog Neuropsychopharmacol Biol Psychiatry 1999; 23(Pt 4): 613624. http://dx.doi.org/10.1016/S0278-5846(99)00020-2

[13] Goldberg MC, Spinellia S, Joela S, Pekara JJ, Dencklaa MB Mostofskya $\mathrm{SH}$. Children with high functioning autism show increased prefrontal and temporal cortex activity during error monitoring. Dev Cogn Neuroscience 2011; 1(Pt 1): 47-56. http://dx.doi.org/10.1016/j.dcn.2010.07.00 
[14] Anagnostou E, Taylor M. Review of neuroimaging in autism spectrum disorders: what have we learned and where do we go from here. Mol Autism 2011; 2(Pt 1): 4. http://dx.doi.org/10.1186/2040-2392-2-4

[15] Just MA, Cherkassky VL, Keller TA, Kana RK, Minshew NJ. Functional and anatomical cortical underconnectivity in autism: Evidence from an FMRI study of an executive function task and corpus callosum morphometry. Cereb Cortex 2007; 17(Pt 4): 951-961.

[16] Lopez BR, Lincoln AJ, Ozonoff S, Lai Z. Examining the relationship between executive functions and restricted, repetitive symptoms of autistic disorder. J Autism Dev Disord 2005; 35: 445-460.

[17] Mosconi MW, Kay M, D'Cruz AM, Seidenfeld A, Guter S, Standford LD, et al. Impaired inhibitory control is associated with higher-order repetitive behaviors in autism spectrum disorders. Psychol Med 2009; 39(Pt 9): 1559-1566. http://dx.doi.org/10.1017/S0033291708004984

[18] South M, Ozonoff S, McMahon WM. The relationship between executive functioning, central coherence and repetitive behaviors in the high-functioning autism spectrum. Autism 2007; 11: 437. http://dx.doi.org/10.1177/1362361307079606

[19] Pierce K, Courchesne E. Evidence for a cerebellar role in reduced exploration and stereotyped behavior in autism. Biol Psychiatry 2001; 49: 655-664.

http://dx.doi.org/10.1016/S0006-3223(00)01008-8

[20] Hardan AY, Girgis RR, Lacerda ALT, Yorbik O, Kilpatrick M, Keshavan $\mathrm{M}$, et al. Magnetic resonance imaging study of the orbitofrontal cortex in autism. J Child Neurol 2005; 21: 866871. http://dx.doi.org/10.1177/08830738060210100701

[21] Arnott B, McConachie H, Meins E, Fernyhough C, Couteur $\mathrm{AL}$, Turner $\mathrm{M}$, et al. The frequency of restricted and repetitive behaviors in a community sample of 15 -month-old-infants. J Dev Behav Pediatr 2010; 3: 223-229. http://dx.doi.org/10.1097/DBP.0b013e3181d5a2ad

[22] Esposito G, Pasca SP. Motor abnormalities as a putative endophenotype for autism spectrum disorders. Front Integr Neurosci 2013; 7(Pt 43): 1-5.

http://dx.doi.org/10.3389/fnint.2013.00043

[23] Nordahl CW, Dierker D, Mostafavi I, Schumann CM, Rivera $\mathrm{SM}$, Amaral DG, et al. Cortical folding abnormalities in autism revealed by surface-based morphometry. J Neurosci 2007; 27(Pt 43): 11725-11735.

http://dx.doi.org/10.1523/JNEUROSCl.0777-07.2007

[24] Goldman S, O'Briende LM, Filipekf PA, Rapina I, Herbertgh MR. Motor stereotypies and volumetric brain alterations in children with autistic disorder. Res Autism Spect Disor 2013; 7(Pt 1): 82-92. http://dx.doi.org/10.1016/j.rasd.2012.07.005

[25] Boyd BA, Conroy MA, Richmond Mancil G, Nakao T, Alter P. Effects of circumscribed interests on the social behaviors of children with autism spectrum disorders. J Autism Dev Disord 2007; 37: 1555-1561.

[26] Richler J, Bishop SL, Kleinke J, Lord C. Restricted and repetitive behaviors in young children with autism spectrum disorders. J Autism Dev Disord 2007; 37: 73-85.

[27] Estes A, Shaw DWW, Sparks BF, Friedman S, Giedd JN, Dawson G, et al. Basal ganglia morphometry and repetitive behavior in young children with autism spectrum disorder. Autism Res 2011; 4(Pt 3): 212-220.

http://dx.doi.org/10.1002/aur.193

[28] Langen M, Schnack HG, Nederveen H, Bos D, Lahuis BE, de Jonge MV, et al. Changes in the developmental trajectories of striatum in autism. Biol Psychiatry 2009; 66: 327-333. http://dx.doi.org/10.1016/j.biopsych.2009.03.017

[29] Langen $M$, Durston $S$, Staal WG, Palmen SJMC, vanEngeland $H$. Caudate nucleus is enlarged in high- functioning medication-naïve subjects with autism. Biol Psychiatry 2007; 62(Pt 3): 262-266.

http://dx.doi.org/10.1016/j.biopsych.2006.09.040

[30] Herbert MR, Ziegler DA, Deutsch CK, O'Brien LM, Lange N, Bakardjiev A, et al. Dissociations of cerebral cortex, subcortical and white matter volumes in autistic boys. Brain 2003; 126(Pt 5): 1182-1192.

http://dx.doi.org/10.1093/brain/awg110

[31] Voelbel G, Bates M, Buckman J, Pandina G, Hendren R. Caudate nucleus volume and cognitive performance: Are they related in childhood psychopathology? Biol Psychiatry 2006; 60(Pt 9): 942-950.

http://dx.doi.org/10.1016/j.biopsych.2006.03.071

[32] South M, Ozonof S, McMahon WM. Repetitive behavior profiles in Asperger syndrome and high functioning autism. J Autism Dev Disord 2005; 35(Pt 2): 145-158.

[33] Kringelbach ML, Rolls ET. The functional neuroanatomy of the human orbitofrontal cortex: evidence from neuroimaging and neuropsychology. Prog Neurobiol 2004; 72(Pt 5): 341372.

http://dx.doi.org/10.1016/j.pneurobio.2004.03.006

[34] Evans DW, Lewis MD, Lobust E. The role of the orbitofrontal cortex in normally developing compulsive-like behaviors and obsessive compulsive disorder. Brain Cogn 2004; 55(Pt 1): 220-234. http://dx.doi.org/10.1016/S0278-2626(03)00274-4

[35] Vink $M$, Kahn RS, Raemaekers $M$, van den Heuvel $M$, Boersma M, Ramsey NF. Function of striatum beyond inhibition and execution of motor responses. Hum Brain Mapp 2005; 25: 336-344. http://dx.doi.org/10.1002/hbm.20111

[36] Zanbelt BB, Vink M. On the role of the striatum in response inhibition. PLoS ONE 2010; 5(Pt 11): 1384.

http://dx.doi.org/10.1371/journal.pone.0013848

[37] Balleine BW, Delgado MR, Hikosaka, O. The role of the dorsal striatum in reward and decision-making. $J$ Neurosci 2007; 27(Pt 31): 8161-8165.

http://dx.doi.org/10.1523/JNEUROSCI.1554-07.2007

[38] Villablanca JR. Why do we have a caudate nucleus? Acta Neurobiol Exp (WARS) 2010; 70: 95-105.

[39] Hagmann P, Cammoun L, Gigandet X, Meuli R, Honey CJ, Wedeen Van J, et al. Mapping the structural core of human cerebral cortex. PLoS Biol 2008; 6(Pt 7): e159. http://dx.doi.org/10.1371/journal.pbio.0060159

[40] Di X, Rao H. Progress in functional connectivity analysis. Progress in Biochemistry and Biophysics 2007; 1: 34-35.

[41] Agam Y, Joseph RM, Barton JJS, Manoach DS. Reduced cognitive control of response inhibition by the anterior cingulate cortex in autism spectrum disorders. Neurolmage 2010; 52: 336-347. http://dx.doi.org/10.1016/j.neuroimage.2010.04.010

[42] Kana RK, Keller TA, Minsher NJ, Just MA. Inhibitory control in high-functioning autism: Decreased activation and underconnectivity in inhibition networks. Biol Psychiatry 2007; 62(Pt 3): 198-206.

http://dx.doi.org/10.1016/j.biopsych.2006.08.004

[43] Schmitz N, Rubia K, Daly E, Smith A, Williams S, Murphy DGM. Neural correlates of executive function in autistic spectrum disorders. Biol Psychiatry 2006; 59: 7-16. http://dx.doi.org/10.1016/j.biopsych.2005.06.007

[44] Mizuno A, Villalobosa ME, Daviesa MM, Dahla BC, Muller R. Partially enhanced thalamocortical functional connectivity in autism. Brain Res 2006; 1104: 160-174.

http://dx.doi.org/10.1016/j.brainres.2006.05.064

[45] Turner KC, Frost L, Linsencardt D, Mcllroy JR, Muller R. Atypical diffuse functional connectivity between caudate nuclei and cerebral cortex in autism. Behav Brain Funct 2006; 2(Pt 1): 34.

http://dx.doi.org/10.1186/1744-9081-2-34 
[46] Boyd BA, McBee M, Holtzclaw T, Baranek GT, Bodfish JW. Relationships among repetitive behaviors, sensory features, and executive functions in high functioning autism. Res Autism Spect Disord 2009; 3(Pt 4): 959-966. http://dx.doi.org/10.1016/j.rasd.2009.05.003

[47] Bolling DZ, Pitskel NB, Deen B, Crowley MJ, McPartland JC, Kaiser MD, et al. Enhanced neural responses to rule violation in children with autism: A comparison to social exclusion. Dev Cogn Neurosci 2011; 1 (Pt 3): 280-294. http://dx.doi.org/10.1016/j.dcn.2011.02.002

[48] Solomon M, Ozonoff SJ, Ursu S, Ravizza S, Cummings N, Ly $S$, Carter CS. The neural substrates of cognitive control deficits in autism spectrum disorder. Neuropsychologia 2009; 47: 2515-2526.

http://dx.doi.org/10.1016/j.neuropsychologia.2009.04.019

[49] Uddin LQ, Supekar K, Lynch CJ, Khouzam A, Phillips J, Feinstein $C$, et al. Salience network-based classification and prediction of symptom severity in children with autism. JAMA Psychiatry 2013; 1-11. http://dx.doi.org/10.1001/jamapsychiatry.2013.104

[50] Paulus MP, Stein MB. An insular view of anxiety. Biol Psychiatry 2006; 60: 383-387. http://dx.doi.org/10.1016/j.biopsych.2006.03.042

[51] Ptak R. The frontoparietal attention network of the human brain: action, saliency, and a priority map of the environment. Neuroscientist 2012; 18(Pt 5): 502-515. http://dx.doi.org/10.1177/1073858411409051

[52] Fan J. Attentional network deficits in autism spectrum disorders. In: Buxbaum, JD and Hof PR, editors. The Neuroscience of Autism Spectrum Disorders. Elsevier Inc 2013, p. 281-288.

[53] Clery H, Andersson F, Bonnet-Brilhault F, Philippe A, Wicker $\mathrm{B}$, Gornot M. fMRI investigation of visual change detection in adults with autism. Neuroimage Clin 2013; 2: 303-312. http://dx.doi.org/10.1016/..nicl.2013.01.010

[54] Shafritz KM, Dichter GS, Baranek GT, Belger A. The neural circuitry mediating shifts in behavioral response and cognitive set in autism. Biol Psychiatry 2008; 63(Pt 10): 974980.

http://dx.doi.org/10.1016/j.biopsych.2007.06.028

[55] Casico JC, Foss-Feig JH, Heacock J, Schauder KB, Loring WA, Rogers BP, et al. Affective neural response to restricted interests in autism spectrum disorders. J Child Psychol Psychiatry 2013; 55(Pt 2): 162-171. http://doi.org/10.1111/jcpp.12147

[56] Dichter GS, Felder JN, Green SR, Rittenberg AM, Sasson NJ, Bodfish JW. Reward circuitry function in autism spectrum disorders. SCAN 2012; 7: 160-172. http://dx.doi.org/10.1093/scan/nsq095

[57] Sabatino A, Rittenberg A, Sasson NJ, Turner-Brown L, Bodfish JW, Dichter GS. Functional neuroimaging of social and nonsocial cognitive control in autism. J Autism Dev Disord 2013; 43(Pt 12): 2903-2913. http://dx.doi.org/10.1007/s10803-013-1837-4

[58] Courchesne E, Townsend J, Akshoomoff NA, Saitoh O, Yeung-Courchesne R, Lincoln AJ, et al. Impairment in shifting attention in autistic and cerebellar patients. Behav Neurosci 1994; 108: 848-856.

http://dx.doi.org/10.1037/0735-7044.108.5.848

[59] Delmonte S, Gallagher L, O'Hanlon E, McGrath J, Balsters $\mathrm{JH}$. Functional and structural connectivity of frontostriatal circuitry in autism spectrum disorder. Front Hum Neurosci 2013; 7 (Pt 430): 1-14

http://dx.doi.org/10.3389/fnhum.2013.00430

[60] McKeown MJ, Jung TP, Makeig S, Brown G, Kindermann SS, Lee T, Sejnowski TJ. Spatially independent activity patterns in functional MRI data during the Stroop colornaming task. Proc Natl Acad Sci USA 1998; 95: 803-810. http://dx.doi.org/10.1073/pnas.95.3.803

[61] Boyd BA, McDonough SG, Bodfish JW. Evidence-based behavioral interventions for repetitive behaviors in autism. $J$ Autism Dev Disord 2012; 42: 1236-1248. http://dx.doi.org/10.1007/s10803-011-1284-z

[62] Biswal BB, Mennes M, Zuo X, Gohel S, Kelly C, Smith SM, et al. Toward discovery science of human brain function. PNAS 2010; 107(Pt 10): 4734-4739. http://dx.doi.org/10.1073/pnas.0911855107

[63] Monk CS, Peltier SJ, Wiggins JL, Weng S, Carrasco M, Risi $\mathrm{S}$, Lord C. Abnormalities of intrinsic functional connectivity in autism spectrum disorders. Neuroimage 2009; 47: 764-772. http://dx.doi.org/10.1016/j.neuroimage.2009.04.069

[64] Weng SJ, Wiggins JL, Peltier SJ, Carrasco M, Risi S, Lord C. Alterations of resting state functional connectivity in the default network in adolescents with autism spectrum disorders. Brain Res 2010; 1313: 202-214. http://dx.doi.org/10.1016/j.brainres.2009.11.057

[65] Stieltjes B, Kaufmann WE, van Zijl PC, Fredericksen K, Pearlson GD, Solaiyappan $M$ et al. Diffusion tensor imaging and axonal tracking in the human brainstem. Neuroimage 2001; 14: 723-735. http://dx.doi.org/10.1006/nimg.2001.0861

[66] Thomas C, Humphreys K, Jung K, Minshew N, Behrmann M. The anatomy of the callosal and visual association pathways in high-functioning autism: a DTI tractography study. Cortex 2011; 47(Pt 7): 863-873 http://dx.doi.org/10.1016/j.cortex.2010.07.006

[67] Thakkar KN, Polli FE, Joseph R, Tuch D, Hadjikhani N, Barton J, et al. Response monitoring, repetitive behavior and anterior cingulate abnormalities in autism spectrum disorders. Brain 2008; I3I: 2464-2478. http://dx.doi.org/10.1093/brain/awn099

[68] Zikopolous B, Barbas $\mathrm{H}$. Changes in prefrontal axons may disrupt the network in autism. J Neurosci 2010; 30(Pt 44): 14595-14609. http://dx.doi.org/10.1523/JNEUROSCI.2257-10.2010

[69] Langen M, Leemans A, Johnston P, Ecker C, Daly E, Murphy $\mathrm{CM}$, et al. Frontal striatal circuitry and inhibitory control in autism: Findings from diffusion tensor imaging tractography. Cortex 2012; 48: 183-193. http://dx.doi.org/10.1016/j.cortex.2011.05.018

\section{DOI: http://dx.doi.org/10.6000/2292-2598.2015.03.01.4}

(C) 2015 Traynor and Hall; Licensee Lifescience Global.

This is an open access article licensed under the terms of the Creative Commons Attribution Non-Commercial License (http://creativecommons.org/licenses/by-nc/3.0/) which permits unrestricted, non-commercial use, distribution and reproduction in any medium, provided the work is properly cited. 